\title{
Sequential Induction of Chirality in Helical Polymers: From the Stereocenter to the Achiral Solvent
}

Belén Nieto-Ortega, Rafael Rodríguez, Samara Medina, Emilio Quiñoá, Ricardo Riguera, Juan Casado, Félix Freire, Francisco Javier Ramírez

\section{Accepted Manuscript}

This is the Accepted Manuscript version of a Published Work that appeared in final form in The Journal of the Physical Chemistry Letters, Copyright (C) 2018 American Chemical Society after peer review and technical edityng by the publisher. To access the final edited and published work see: https://pubs.acs.org/doi/10.1021/acs.jpclett.8b00519

\section{How to cite:}

Nieto-Ortega, B., Rodríguez, R., Medina, S., Quiñoá, E., Riguera, R., \& Casado, J. et al. (2018). Sequential Induction of Chirality in Helical Polymers: From the Stereocenter to the Achiral Solvent. The Journal Of Physical Chemistry Letters, 9(9), 2266-2270. doi: 10.1021/ acs.jpclett.8b00519

\section{Copyright information:}

(C) 2018 American Chemical Society 


\section{Sequential Induction of Chirality in Helical \\ Polymers: from the Stereocenter to the Achiral}

\section{Solvent}

Belén Nieto-Ortega, ${ }^{\dagger}$ Rafael Rodríguez, ${ }^{\S}$ Samara Medina, ${ }^{\#}$ Emilio Quiñoá, ${ }^{\S}$ Ricardo Riguera, ${ }^{\S}$ Juan Casado, ${ }^{\#}$ Félix Freire ${ }^{*, \S}$ and Francisco Javier Ramírez, ${ }^{*, \#}$

${ }^{\dagger}$ IMDEA Nanoscience. Ciudad Universitaria de Cantoblanco. Faraday 9, 28049 Madrid (Spain)

${ }^{\S}$ Centro Singular de Investigación en Química Biolóxica e Materials Moleculares (CIQUS) and Departamento de Química Orgánica, Universidade de Santiago de Compostela, 15782 Santiago de Compostela (Spain).

\# Departamento de Química Física, Facultad de Ciencias, Universidad de Málaga, Campus de Teatinos s/n, 29071 Málaga (Spain). 
ABSTRACT. Several steps of chiral induction have been detected in poly(phenylacetylene)s among their different hierarchical levels of chirality by vibrational circular dichroism, namely: i) from the stereogenic centers to the innermost polyacetylene helical covalent backbone (helix ${ }_{\text {int }}$ ); ii) from this to the external helix (helix $\mathrm{ext}_{\text {) }}$ formed by the side phenyl pendants which forms a complementary helix or counter-helix, and iii) from this pendant helix to the helical solvation sphere (helix $\mathrm{x}_{\text {solv. }}$ ), being the last one observed along this work. The pendant to polyene backbone chiral induction determines the helical structure adopted by the polymer, and therefore the solvation helix. This helical structure is promoted by two mechanisms: steric effects and hydrogen bonding. An important finding concerns the demonstration by VCD of how an achiral solvent becomes chirally organized owing to the template effect of the covalent polymer helices, an effect that is silent to other structural techniques such as ECD or AFM and that hence significantly broadens the scope of these previous analyses.

\section{TOC GRAPHICS}

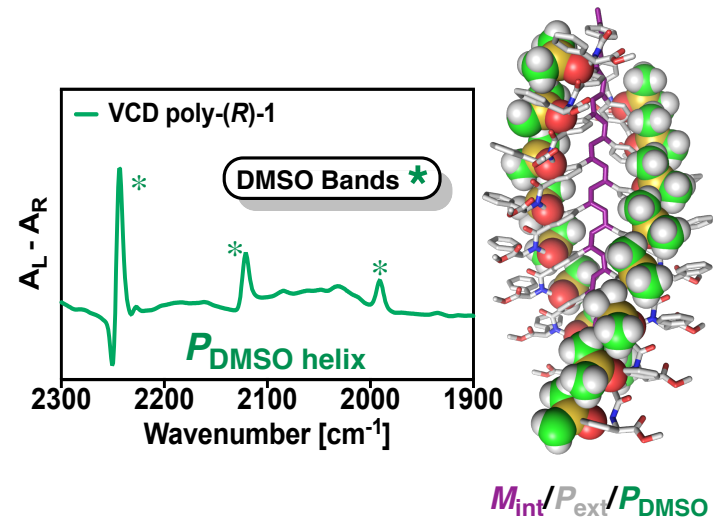


The term chirality is used to designate those objects that cannot be superimposable on its mirror image. ${ }^{1}$ In Chemistry, chirality is a molecular characteristic which causes the appearance of unique properties, ${ }^{2-3}$ related with fundamental topics as chemical reactivity, biological activity or light interaction. Molecular chirality can be triggered by different structural motifs. The primary cause is the presence of a stereocenter, or intrinsic chirality, usually in the form of an asymmetric carbon. ${ }^{4}$ In macromolecules and molecular assemblies the main chiral motif is the helix which can exist as two different enantiomers, right-handed or clockwise ( $P$ type) and lefthanded or anti-clockwise ( $M$ type), which coexist at $50 \%$ each when the constituent units are achiral. When a chirality source, either intrinsic or extrinsic, is present, a dominant helix handedness prevails and chiro-optical activity emerges. The molecular helices can also selfassemble forming double-helices, super-helices, etc., or can be used as chiral seeds where other molecules template with a pre-established chirality sign. ${ }^{5-7}$ In supramolecular assemblies, transfer and induction processes through the hierarchical levels of chiral organization is a key aspect of their dynamics. ${ }^{8-15}$

Herein, we will demonstrate the chirality induction in poly(phenylacetylene)s (PPA), ${ }^{16-20}$ laterally functionalized with phenylglycine methyl ester groups (i.e., the pendant groups, Fig. 1). ${ }^{21}$ PPA functionalization with this kind of sterically demanding groups forces the poly(phenylacetylene) to lose its ideal planar all-transoid shape resulting in complex helical-type structures whose handedness is dictated by the chiral seed of the pendants $[(R)$ - or $(S)-] .{ }^{20}$ In this work, we afford a full assessment of the interconnection between stereocenter and helix sources of chirality and the action of these polymers as chiral templates of other supramolecular structures with inherited chiral properties. To perform these studies we have used vibrational 
circular dichroism (VCD), the chiral branch of the infrared spectroscopy, which has been successfully applied to structural studies in other polymers. ${ }^{22-24}$

a)<smiles>CC=C(C)Cc1ccc(C(N)=O)cc1</smiles><smiles>COC(=O)C(N)c1ccccc1</smiles>

Poly-(S)-1<smiles>CC(C)=CC(C)C</smiles>

b)

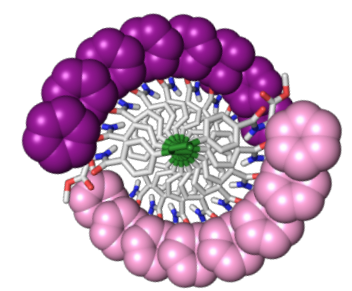

Clockwise Pendants<smiles>COC(=O)[C@H](NC(=O)O)c1ccccc1</smiles>

Poly-(R)-1

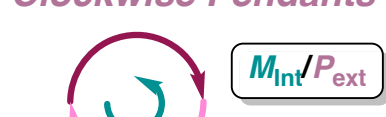

Counterclockwise Backbone

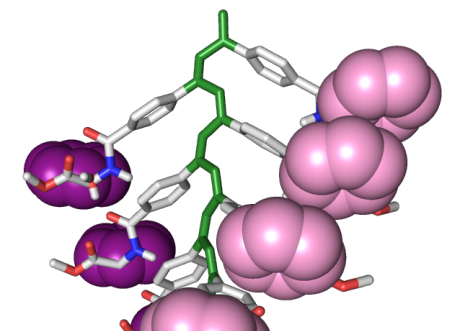

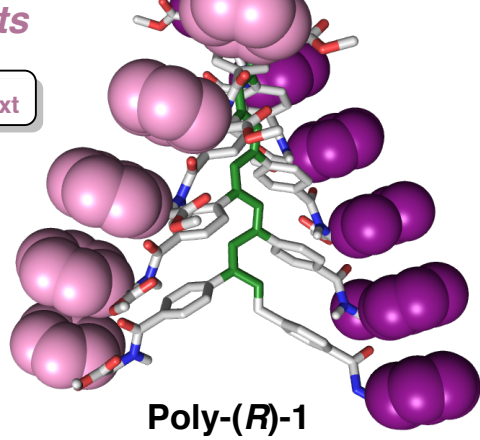

Poly-(R)-1

Figure 1. a) Chemical structures of poly- $(R)-1$ and poly-(S)-1. b) 3-D model of poly- $(R)-1$ in polar solvent $\left(P_{\text {int }} / M_{\text {ext }}\right)$.

Fig. 2 shows the infrared, VCD, electronic absorption and electronic circular dichroism (ECD) spectra of poly- $(R)-\mathbf{1}$ and poly-(S)-1. The two polymers exhibit identical electronic absorption spectra and perfect ECD mirror-images. Recently, TD/DFT calculations on PPA oligomers have demonstrate that the first Cotton effect around $380 \mathrm{~nm}$ is assigned to a $\pi \rightarrow \pi^{*}$ excitations the vinyl moiety. ${ }^{25}$ Thus, the ECD spectra evidence the effective chiral induction from the phenylglycine stereogenic unit to the innermost backbone, whose helix sense is controlled by the $(R)$ - or $(S)$ - configuration of the asymmetric carbons. As a consequence, the polyacetylene backbone of poly- $(R)-1$ will adopt a clockwise helix $\left(P_{\text {int }}\right)$, and concomitantly 
poly-(S)-1 turns in the opposite direction $\left(M_{\mathrm{int}}\right){ }^{22-24}$ It has been previously reported that surrounded this backbone helix in poly- $(R)-\mathbf{1}$ and poly- $(S)-\mathbf{1}$, the pendants by steric repulsion are disposed by forming a second peripheral counter-helix regarding the innermost one. ${ }^{17}$ We now provide direct experimental evidence of the formation of this pendant counter-helix.

a)

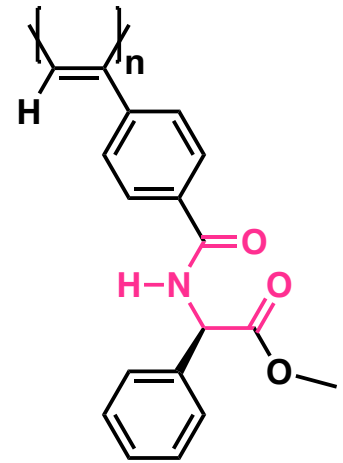

Poly-(R)-1

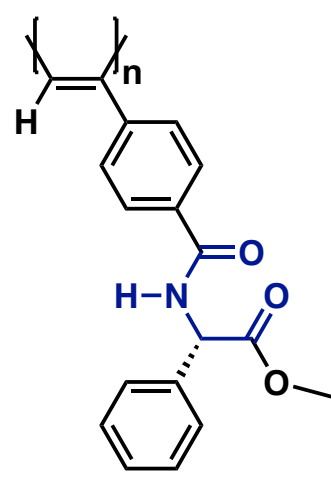

Poly-(S)-1 b)

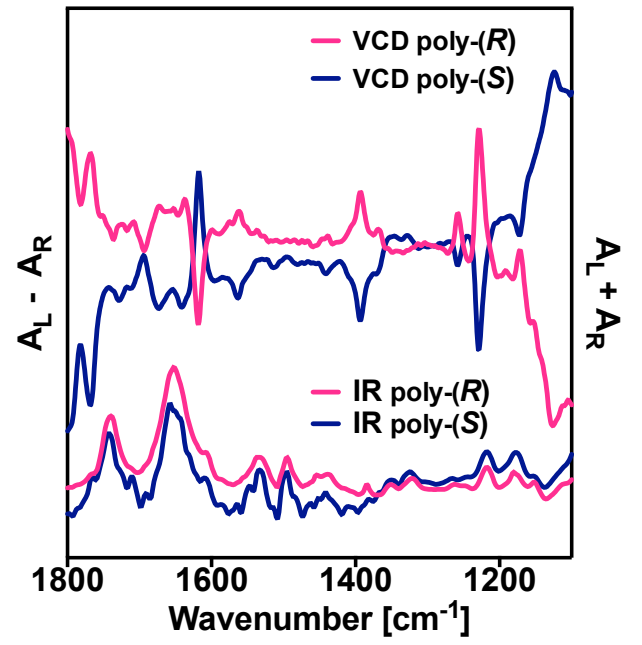

c)

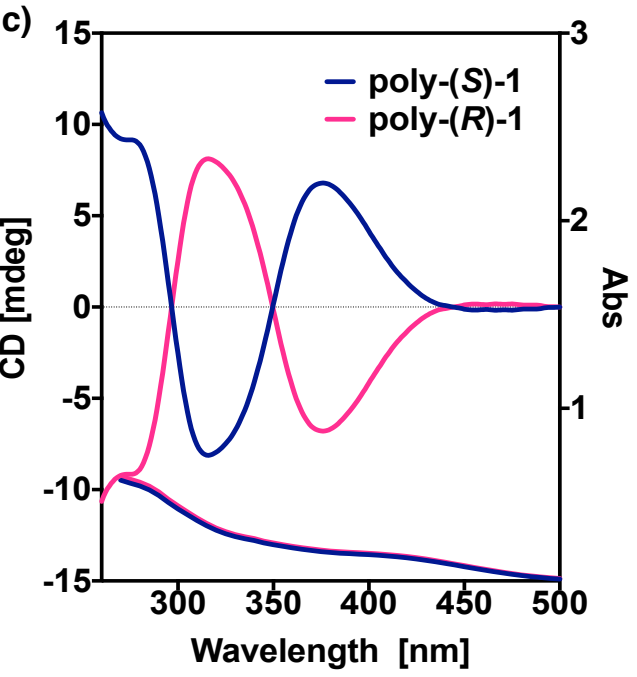

Figure 2. a) The synperiplanar conformations of poly-(R)-1 and poly-(S)-1. b) VCD (top) and infrared (bottom) spectra of poly-(R)-1 (red) and poly-(S)-1 (blue) recorded as DMSO-d 6 solutions c) ECD (top) and electronic absorption (bottom) spectra of poly- $(R)-\mathbf{1}$ (red) and poly- 
(S)-1 (blue) recorded as $0.1 \mathrm{~g} / 1$ DMSO-d 6 solutions. b) VCD (top) and infrared (bottom) as 0.1 $\mathrm{kg} / 1$ DMSO-d solutions. $^{26}$

The infrared absorption spectra of the two polymers are very similar and the corresponding VCD spectra also display great mirror-like images. Both infrared and VCD spectra are dominated by bands belonging to the pendant moieties as these are markedly polar. ${ }^{27-28}$ Interestingly, from the above ECD experiments and reported AFM structural data, we infer that in DMSO poly- $(R)-1$ solution, the stereogenic $(R)$-chiral centre transmits the formation of a $P_{\text {int }}$ helix, whereas it was supposed that the pendant groups should arrange into a $M_{\text {ext }}$ helix. We now verify this by direct VCD detection of the chiroptical response of the external helix. This means that the $(R)$ - configuration at the pendant groups commands a $P$ helix, while the phenylglycine helical arrangement adopts the opposite $M$ chirality. Therefore, the chain of transmission of the chiral information goes through the steric repulsion among the pendants that makes the first transmission step as $(R)$-phenylglycine $\rightarrow P_{\text {int }}$ (pendant absolute configuration to backbone internal helix), and the second one between the two coaxial helices (internal to external helix), $P_{\text {int. }} \rightarrow M_{\text {ext. }}{ }^{22-24}$ In line with this chirality transmission mechanism activated by steric repulsion in poly-1 series, it was found that a helix inversion can be produced by the action of different external stimuli (metal ions, solvents) on the conformational composition at the pendant moiety. ${ }^{20}$

In this regard, we decided to explore if conformational changes at the pendant group produce observable changes in VCD spectra. For this, we conducted theoretical VCD studies in the 1800$1600 \mathrm{~cm}^{-1}$ region for the monomeric unit, $\mathrm{m}-(R)-\mathbf{1}$, varying the $\mathrm{N}-\mathrm{C}-\mathrm{C}-\mathrm{O}$ dihedral angle and maintaining unchanged the rest of geometrical parameters. The VCD spectra over the dihedral scanning, Fig. 3a, show a sign inversion pattern localized between 75 and 90 deg for the Amide I 
band (an invariant pattern is predicted for the carbonyl ester $\mathrm{C}=\mathrm{O}$ stretch) which actually mimics the anti $\rightarrow$ syn conformational change revealing a relationship between the sign of the amide I VCD band and the conformation of the pendant. Hence, if the carbonyl and the amide bands show same VCD signal, both groups are syn oriented, while if the two bands show opposite sign, the two groups are disposed in anti.
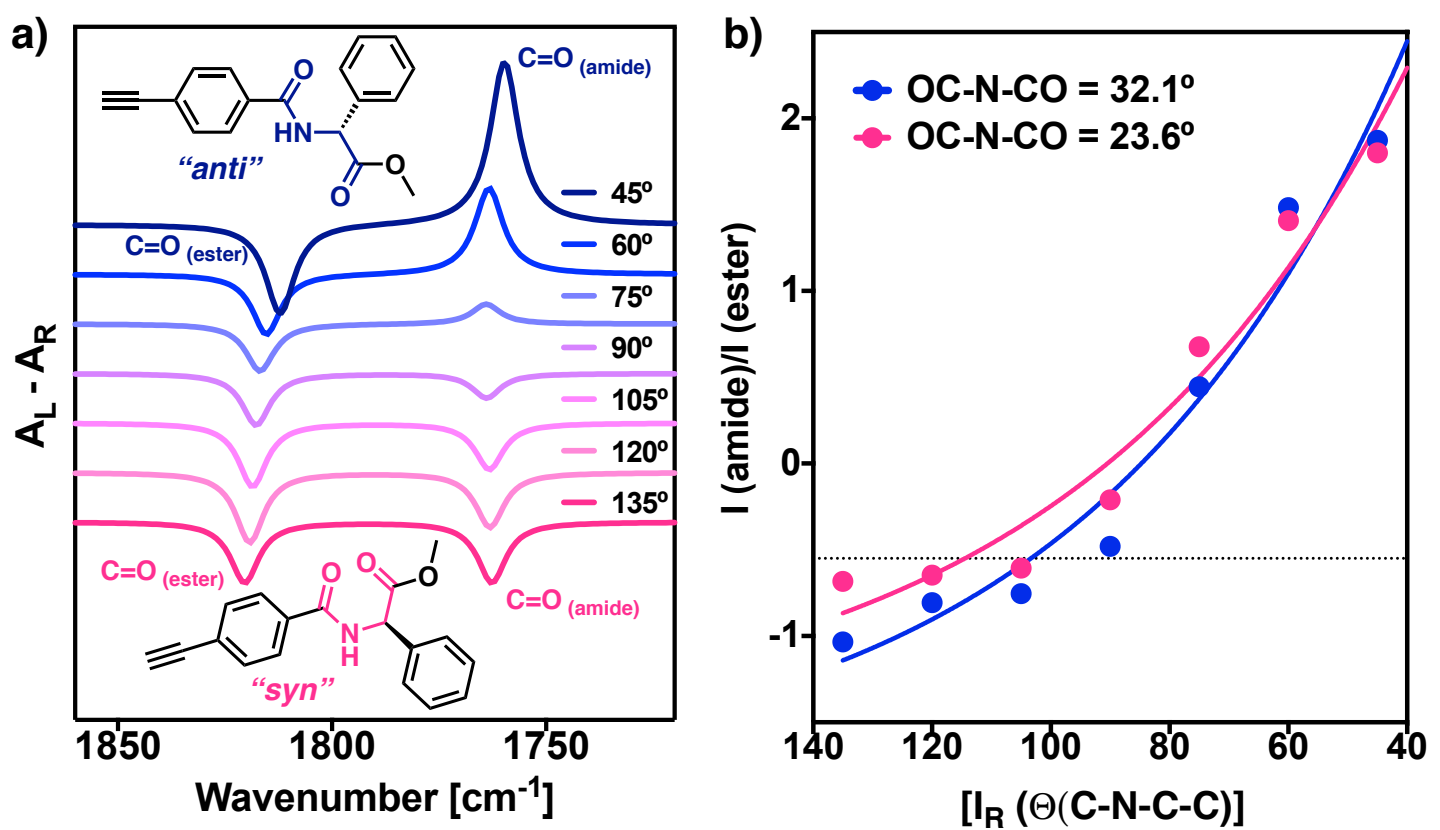

Figure 3. Calculated (CAM-b3lyp/cc-pvtz) spectra of the monomer m- $(R)-\mathbf{1}$ at different N-CC-O dihedral angles. b) Plot of the calculated VCD intensity ratio between the amide I band and the carbonyl stretching vibration of the ester group as a function of the $\mathrm{N}-\mathrm{C}-\mathrm{C}-\mathrm{O}$ dihedral angle of $\mathrm{m}-(R)-\mathbf{1}$. The color indicates the fixed value for the OC-N-C-CO dihedral angle: $23.6 \mathrm{deg}$ (red) and $32.1 \mathrm{deg}$ (blue), which are the calculated dihedrals for the pendant conformation in low/medium polar and highly polar solvents. ${ }^{29}$ The horizontal black line denotes the intensity ratio measured in the VCD experimental spectra of poly-(R)-1 and poly- $(S)-\mathbf{1}$. 
Additionally, in Fig $3 \mathrm{~b}$ we have displayed the intensity ratio $\left(\mathrm{I}_{\mathrm{R}}=\mathrm{I}_{\mathrm{A}} / \mathrm{I}_{\mathrm{E}}\right)$ between the activities of the Amide $\mathrm{I}\left(\mathrm{I}_{\mathrm{A}}\right)$ and of the ester-carbonyl stretching $\left(\mathrm{I}_{\mathrm{E}}\right)$ bands as a function of the C-N-C-C dihedral angle $\left[\mathrm{I}_{\mathrm{R}}(\Theta[\mathrm{C}-\mathrm{N}-\mathrm{C}-\mathrm{C}])\right]$ for two different values of the OC-N-C-CO angles (these angles are taken from the experimental x-ray structure of the monomer). The result shows two similar behaviours for the two starting OC-N-C-CO angles indicating a robust connection between VCD signal and pendant conformation. From our experimental studies, we found that in case of poly- $(R)-1$ dissolved in DMSO, the $\mathrm{I}_{\mathrm{R}} \mathrm{VCD}$ number is -0.55 (dotted line in Fig. $3 b$ ), which corresponds to a C-N-C-C dihedral angle in the 111.3 degrees (red curve) and 100.4 degrees (blue curve) range consistent with a clear syn conformation of the pendant groups. As a result, this clearly indicates that VCD can be used to determine not only the presence of a PPA helical structure, but also to determine the conformation at the pendant group responsible for this helical sense induction.

The VCD spectra of poly-(R)-1, poly-(S)-1 and their corresponding monomers m- $(R)-\mathbf{1}, \mathrm{m}-$ (S)-1, contain surprising information in the region between 1900 and $2400 \mathrm{~cm}^{-1}$, where the bands associated to the carbon-deuterium stretching modes of the DMSO- $\mathrm{d}_{6}$ molecules appear (see Fig. 4 for poly- $(R)-1$ and $\mathrm{m}-(R)-1$ and Fig. S1 in the Supporting Information for the enantiomeric form poly-(S)-1 and m-(S)-1). In principle, the achiral character of the solvent molecules makes them completely VCD inactive. However, these spectra clearly display three strong VCD features measured at the same wavenumbers than the DMSO- $\mathrm{d}_{6}$ infrared absorption bands of the C-D stretching vibrations. Furthermore, the signs of these VCD bands are directly governed by the chirality of the solute, showing a mirror-like behaviour in response to the $(R)-\rightarrow(S)$-absolute configuration change. In addition, these bands are extraordinarily intense in comparison with the 
solute, indicating that plenty of solvent molecules are arrayed into a supramolecular structure surrounding the polymer and the monomer (Fig. 4).

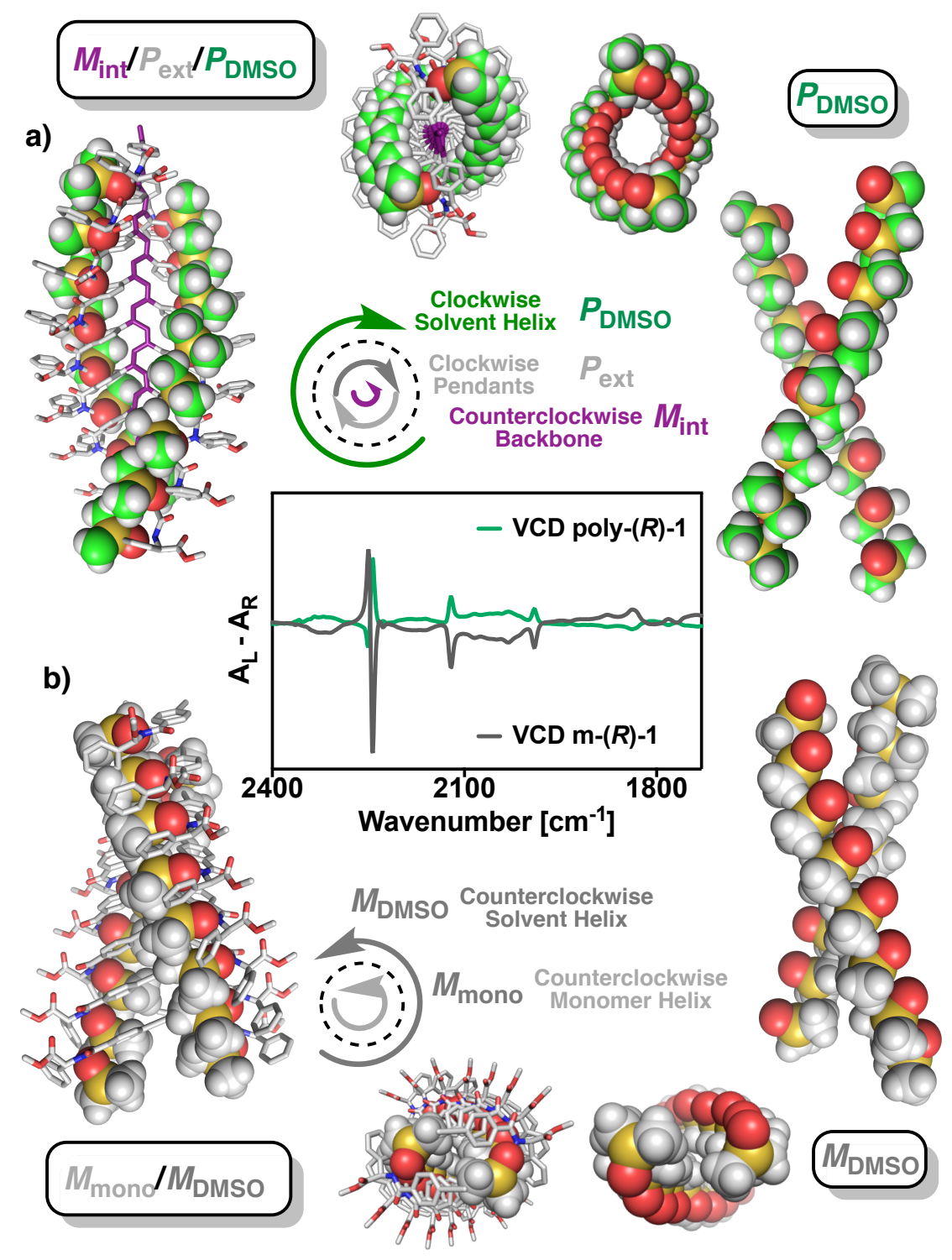

Figure 4. a) VCD spectra of (a) poly-(R)-1 and (b) m-(R)-1 recorded as $0.1 \mathrm{~kg} / 1 \mathrm{DMSO}-\mathrm{d}_{6}$ solutions. Schematic illustration of the helical arrangement of DMSO molecules around (a) poly$(R)-1$ scaffold and (b) m- $(R)-1$ is also shown -DMSO molecules are highlighted in space filling-. 
Interestingly, when the VCD spectra of monomers is compared to their corresponding PPA, $e$. g. m- $(R)-\mathbf{1}$ with poly- $(R)-\mathbf{1}$, opposite VCD profiles are detected in the DMSO region, or, in other words, the spectrum of the $\mathrm{m}-(S)-\mathbf{1}$ matches that of poly- $(R)-1$ polymer whereas that of $\mathrm{m}-(R)-\mathbf{1}$ resembles poly-(S)-1 (see Fig. S2 in the Supporting Information). Clearly, the VCD spectrum is indicating that the solvent networks wrapping both monomer and polymer solutes, are opposite in despite of having the same source of chirality (e.g. m- $(R)-\mathbf{1}$ and poly- $(R)-\mathbf{1}$, Figure 4$)$. Given the absence of any rigid innermost backbone in the monomers, their molecules are free to adopt the natural arrangement dictated by the stereogenic centres (e.g. $R \rightarrow P$ and $S \rightarrow M$ ) and consequently the surrounding solvent molecules get organized according to the supramolecular template described by $\mathrm{m}-(R$ or $S) \mathbf{- 1}$ (Fig. $4 \mathrm{~b})$. On the other hand, in the polymers, the steric hindrances imposed by the pendant group onto the polyacetylene backbone dictates the dihedral angle between conjugated double bond, resulting into the adoption of either a cis-cisoidal or cistransoidal helix. Usually, in a cis-cisoidal helix, the internal helix described by the polyacetylene backbone, and the external one described by the pendant groups rotate in the same direction, while in the case of a cis-transoidal one, the internal and external helices rotate in opposite directions. Accordingly, the solvent molecules will be arrayed describing helical structure where the external helix of the PPA is used as template to pre-organize the solvent molecules (Fig. 4a).

This chiral induction to the achiral solvent represents the main finding of this research. From the beginning, the transfer of chirality between solvents and solutes has received quite attention, although the amount of papers that have used VCD to study the influence of a chiral solute on the nearest solvent shells is significantly low. ${ }^{30-33}$ Here we provide direct observation of a chiroptical response fully localized in an achiral solvent, which can be only explained by the organization of the solvent molecules around the target solutes, thus forming a coordination 
sphere or solvation sphere around them which is able of sensing the solute chirality. Interestingly, the VCD solvent bands are stronger than those of the solute revealing: i) the solvation sphere is arranged in a helical shape provoking an unique VCD enhancement in the sense that a new chirality source (solvent helix) is available; and ii) the organization of the solvent around the solute might expand beyond one coordination sphere to which the chiral effect of the solute is transmitted. In line with this, the DMSO- $\mathrm{d}_{6}$ molecules are able to establish $\mathrm{S}=\mathrm{O} \cdots \mathrm{H}-\mathrm{N}$ hydrogen bonds providing a path and mechanism of intrinsic chiral induction from the pendants to the solvent molecules. In this regard, a model consisting of a 1:1 monomerDMSO hydrogen-bonded adduct has been constructed on which VCD calculations have been carried out. Clearly this model is insufficient to reproduce the intensity of the experimental VCD bands of the solvents (see Fig. S3 in the Supporting Information) that can be justified due to the existence of more than one sphere of coordination.

As a consequence, these studies show that the solute acts as a template of a new helix in the solvent, a solvation helix, whose chirality would be fully controlled by the handedness of the pendant helix. In this model, the chiroptical sign and activity of the solvent molecules are not determined by chiral induction from the asymmetric carbon atoms of the pendants, but from the helix of the pendants (Fig. 4). As a result, different chiral induction events in the polymers occur to produce the chiral amplification of the solvent. These events are described as follows: $R($ stereogenic center $) \rightarrow M($ innermost polyacetylene helix $) \rightarrow P($ external pendant helix $) \rightarrow$ $P($ solvation helix $)$ or $S$ (stereogenic center $) \rightarrow P($ innermost polyacetylene helix $) \rightarrow M($ external pendant helix $) \rightarrow M($ solvation helix), all mediated by two principal mechanisms: the steric effect and the hydrogen bonding. 
The existence of this solvation helix has been investigated in other phenylglycine-PPA derivatives with the chiral $(R)$ - or $(S)$-pendant linked to the meta position of the phenyl group (m$(R)-2, \mathrm{~m}-(S)-2$, poly- $(R)-2$ and poly-(S)-2, see Figs. S4 and S5 in the Supporting Information). The different aromatic substitution pattern could modify the chiral transmission chain as it also might modify the steric interactions between pendants. However, it is known that the correlation between the enantiomeric form and the helix handedness is the same to the one existing in poly-1 [poly-(R)-2, $P_{\text {int }} / M_{\text {ext }}$; poly-(S)-2, $M_{\text {int }} / P_{\text {ext }}$. Therefore, we observe as expected that the VCD spectra of poly-(R)-2 and poly-(S)-2 exhibit similar patterns to their respective counterparts poly(R)-1 and poly- $(S)-\mathbf{1}$.

In order to get further insight about the experimental observation of opposite VCD profiles for polymers and monomers with the same stereocenter, molecular mechanics (MM) simulations taking the monomer samples were performed. Two different 18-mer propellers of the two enantiomers, a CW, $P$ sense, and a $\mathrm{CCW}, M$ sense, were stabilized in a polar (DMSO-d 6 ) and non-polar (hexane) environments (see Fig. S6 in the Supporting Information). The predicted stabilization energies, showing the relative stability of our 18-mer helix, are listed in Table 1. An anti conformation, which allows the amide group to form hydrogen bonds between the monomers of the helix, is deduced for $\mathrm{m}-(R)-\mathbf{1}$ in a non-polar environment. On the other hand, a syn conformation is observed in a polar environment. In this conformation no hydrogen bonds are available between the monomers with the polar groups directed to the solvent ambient instead of being oriented regarding the vicinal monomer (see Fig. S6). Additionally, the monomer helices are energetically sustained by $\pi$ - $\pi$ stacking between the benzene rings, which thus replace the polyacetylene backbone as the initial seed upon which subsequent chiral organizations take place. In this case, the helical structure described by the monomer 
supramolecular aggregation is directly dependent on the absolute configuration of the phenylglycine. Moreover, the solvent will be rearranged wrapping the monomeric supramolecular aggregate, generating a helical structure with the same helical sense than the corresponding monomer helix. Thus, $\mathrm{m}-(R)-\mathbf{1}$ in a polar solvent generates a $M$ helix that serves as template to generate a $M_{\text {solvent }}$ helix, producing a chiral amplification of its VCD bands with a negative sign (Figure 4b). On the other hand, poly- $(R)-\mathbf{1}$ shows again chiral amplification on the DMSO VCD bands. In this case the sign of the VCD bands are positive, opposite to the ones obtained for m-(R)-1, which a can be easily explained due to the presence of a $P_{\text {ext }}$ helix, described by the pendant groups, which serve as template to generate a $P_{\text {solvent }}$ helix (Figure $4 \mathrm{a}$ ).

Table 1. Stabilization energies per monomer $(\mathrm{kcal} / \mathrm{mol})$ of the $18-\mathrm{mer}$ double helices of $\mathrm{m}$ $(R)-\mathbf{1}$ and $\mathrm{m}-(S)-\mathbf{1}$, using either hexane and DMSO- $\mathrm{d}_{6}$ as continuum solvation models The values were calculated by subtracting the energy of an isolated monomer to the energy of the oligomer divided by 18 .

\begin{tabular}{lllll}
\hline Solvent & DMSO-d $_{6}$ & \multicolumn{3}{c}{ Hexane } \\
\hline Helix handedness & $\mathrm{CCW} / P$ & $\mathrm{CW} / M$ & $\mathrm{CCW} / P$ & $\mathrm{CW} / M$ \\
\hline $\mathrm{m}-(R)-\mathbf{1}$ & 14.99 & 17.67 & 8.26 & 7.43 \\
\hline $\mathrm{m}-(S)-\mathbf{1}$ & 15.31 & 13.20 & 9.38 & 9.88 \\
\hline
\end{tabular}

Summarizing, we have reported VCD experimental evidence of the chiral induction event in functionalized poly(phenylacetylene)s throughout their different hierarchical levels and, 
noteworthy, chiral induction on the DMSO solvent was observed due to the formation of a solvation helix around the helical PPA. The high relative intensity of the solvent bands in relation to the PPA bands indicates the presence of a chiral enhancement phenomenon produced by the presence of a solvent helical macrostructure. A similar effect was observed in DMSO solutions of the monomeric units, which resulted in opposite helical sense to the one observed in the polymer with identical enantiomeric form $[(R)$ - or $(S)$-]. Thus, we have reported how chirality, in particular conditions, is uniquely amplified and transmitted among different levels, bottom-up hierarchical transmission of chirality, or transmission from local stereogenic centers to covalent helical structures to supramolecular helices in the solvents. This, in our opinion, constitutes a nice proof-of-concept of chiral template of an achiral environment, where the chiral enhanced signal of the solvent can be indirectly used to determine an excess of a single-handed helix in the PPA.

\section{ASSOCIATED CONTENT}

Supporting Information. Experimental and computational details, VCD spectra of poly-(S)-1 and $\mathrm{m}-(S)-\mathbf{1}$ in DMSO- $\mathrm{d}_{6}$, infrared and VCD spectra of the monomers $\mathrm{m}-(R)-\mathbf{1}$ and $\mathrm{m}-(S)-\mathbf{1}$ in solid state and in DMSO- $\mathrm{d}_{6}$ solution, Calculated VCD spectra of monomer-DMSO hydrogen bonding 1:1 complexes, vibrational and electronic spectra of poly- $(R)-\mathbf{2}$ and poly- $(S)-2$ in DMSO- $\mathrm{d}_{6}$, and models of clockwise $(\mathrm{CW}-M)$ and counterclockwise $(\mathrm{CCW}-P) \mathrm{m}-(R)-\mathbf{1}$ helices in polar (DMSO) and non-polar (hexane) environments. 


\section{AUTHOR INFORMATION}

\section{Corresponding Authors}

F. Freire: felix.freire@usc.es

F.J.Ramírez: ramirez@uma.es

\section{Notes}

The authors declare no competing financial interests.

\section{ACKNOWLEDGMENT}

Financial support from Ministerio de Ciencia e Innovación [CTQ2014-61470-EXP, CTQ2015-

69391-P, FPI (R. Rodríguez), FPU (S. Medina), Juan de la Cierva postdoctoral Fellowship FJCI2015-23531 (B.Nieto-Ortega)], Xunta de Galicia (GRC2014/040, Centro singular de investigación de Galicia accreditation 2016-2019, ED431G/09) and the European Regional Development Fund (ERDF) is gratefully acknowledged. 


\section{REFERENCES}

(1) Kelvin, W.T. Baltimore Lectures on Molecular Dynamics and the Wave Theory of Light, CJ Clay, London, UK, 1904.

(2) Pasteur, L. Transformation des Acides Tartriques en Acide Racémique. Découverte de l'Acide Tartrique Inactif. Nouvelle Méthode de Séparation de l'Acide Racémique en Acides Tartriques Droit et Gauche. C.R. Acad. Sci. Paris 1863, 37, 162-166.

(3) Busch, K.W.; Busch, M.A. (eds). Chiral Analysis, Elsevier, Amsterdam, The Netherlands, 2006.

(4) Cahn, R.S.; Ingold, C.; Prelog, V. Specification of Molecular Chirality. Angew. Chem. Int. Ed. 1966, 5, 385-415.

(5) Hadjichristidis, N.; Hirao, A.; Tezuka, Y.; Prez, F.D. Complex Macromolecular Architectures: Synthesis, Characterization, and Self Assembly, John Wiley and Sons (Asia), Singapore, 2011.

(6) Harada, A. Supramolecular Polymer Chemistry, Wiley-VCH, Weinheim, Germany, 2012.

(7) Dequan, A.L. Molecular Self-Assembly: Advances and Applications, CRC Press, Boca Raton, FL, 2013.

(8) Yashima, E.; Ousaka, N.; Taura, D.; Shimomura, K.; Ikai, T.; Maeda, K. Helical Systems: Helical Assemblies of Small Molecules, Foldamers, and Polymers with Chiral Amplification and Their Functions. Chem. Rev. 2016, 116, 13752-13990.

(9) Yu, Z.; Hecht, S. Remote Control Over Folding by Light. Chem. Commun., 2016, 52, $6639-6653$. 
(10)Le Bailly, B.A.F.; Clayden, J. Dynamic Foldamer Chemistry. Chem. Commun., 2016, $52,4852-4863$.

(11) Schwartz, E.; Koepf, M.; Kitto, H. J.; Nolte, R. J. M.; Rowan, A. E. Helical Poly(isocyanides): Past, Present and Future. Polym. Chem., 2011, 2, 33-47.

(12)Iida, H.; Yashima, E. Synthesis and Application of Helical Polymers with Macromolecular Helicity Memory, in Polymeric Chiral Catalyst Design and Chiral Polymer Synthesis, ed. S. Itsuno, John Wiley \& Sons, Hoboken, NJ, USA, 2011, ch. 7, p. 201.

(13)Rosen, B. M.; Wilson, C. J.; Wilson, D. A.; Peterca, M.; Imam, M. R.; Percec, V. Dendron-Mediated Self-Assembly, Disassembly, and Self-Organization of Complex Systems. Chem. Rev., 2009, 109, 6275-6540.

(14) Yashima, E.; Maeda, K.; Iida, H.; Furusho, Y.; Nagai, K. Helical Polymers: Synthesis, Structures, and Functions. Chem. Rev., 2009, 109, 6102-6211.

(15)Rudick, J. G.; Percec, V. Induced Helical Backbone Conformations of Self-Organizable Dendronized Polymers. Acc. Chem. Res., 2008, 41, 1641-1652.

(16) Arias, S.; Freire, F.; Calderón, M.; Bergueiro, J. Unexpected Chiro-Thermoresponsive Behavior of Helical Poly(phenylacetylene)s Bearing Elastin-Based Side Chains. Angew. Chem. Int. Ed., 2017, 56, 11420-11425.

(17)Freire, F.; Quiñoá, E.; Riguera, R. Chiral Nanostructure in Polymers Under Different Deposition Conditions Observed Using Atomic Force Microscopy of Monolayers: Poly(phenylacetylene)s as a Case Study. Chem. Commun., 2017, 53, 481-492. 
(18) Van Leeuwen, T.; Heideman, H. G.; Zhao, D.; Wezenberg, S. J.; Feringa, B. L. In Situ Control of Polymer Helicity with a Non-Covalently Bound Photoresponsive Molecular Motor Dopant. Chem. Commun., 2017, 53, 6393-6396.

(19)Wang, S.; Chen, J.; Feng, X.; Shi, G.; Zhang, J.; Wan, X. Conformation Shift Switches the Chiral Amplification of Helical Copoly(phenylacetylene)s from Abnormal to Normal “Sergeants-and-Soldiers” Effect. Macromolecules, 2017, 50, 46104615.

(20)Freire, F.; Quiñoá, E.; Riguera, R. Supramolecular Assemblies from Poly(phenylacetylene)s. Chem.Rev., 2016, 116, 1242-1271.

(21)Rodríguez, R.; Quiñoá, E.; Riguera, R. Freire, F. Architecture of Chiral Poly(phenylacetylene)s: From Compressed/Highly Dynamic to Stretched/Quasi-Static Helices. J. Am. Chem. Soc. 2016, 138, 9620-9628.

(22)Hase, Y.; Nagai, K.; Iida, H.; Maeda, K.; Ochi, N.; Sawabe, K.; Sakajiri, K.; Okoshi, K.; Yashima, E. Mechanism of Helix Induction in Poly(4-carboxyphenyl Isocyanide) with Chiral Amines and Memory of the Macromolecular Helicity and Its Helical Structures. $J$. Am. Chem. Soc. 2009, 131, 10719-10732.

(23)Kawauchi, T.; Kumaki, J.; Kitaura, A.; Okoshi, K.; Kusanagi, H.; Kobayashi, K.; Sugai, T.; Shinohara, H; Yashima, E. Encapsulation of Fullerenes in a Helical PMMA Cavity Leading to a Robust Processable Complex with a Macromolecular Helicity Memory. Angew. Chem. Int. Ed. 2008, 47, 515-519.

(24)Tang, H. Z.; Novak, B. M.; He, J.; Polavarapu, P. L. A Thermal and Solvocontrollable Cylindrical Nanoshutter Based on a Single Screw-Sense Helical Polyguanidine. Angew. Chem. Int. Ed. 2005, 44. 7298-7301. 
(25)Fernández, B.; Rodríguez, R.; Rizzo, A.; Quiñoña, E.; Riguera, R.; Freire, F. Predicting the Helical Sense of Poly(phenylacetylene)s from their Electron Circular Dichroism Spectra. Angew. Chem. Int. Ed. 2018, 49. DOI: 10.1002/ anie.201713164.

(26)Wang, F.; Polavarapu, P.L.; Lebon, F.; Longhi, G.; Abbate, S.; Catellani, M. Absolute Configuration and Conformational Stability of (S)-(+)-3-(2-Methylbutyl)thiophene) and (+)-3,4-Di[(S)-2-methylbutyl)]thiophene and Their Polymers. J. Phys. Chem. A 2002, $106,5918-5923$.

(27)Ma, S.; Cao, X.; Mak, M.; Sadik, A.; Walkner, C.; Freedman, T.B.; Lednev, I.K.; Dukor, R.K.; Nafie, L.A.. Vibrational Circular Dichroism Shows Unusual Sensitivity to Protein Fibril Formation and Development in Solution. J. Am. Chem. Soc. 2007, 129, 1236412365.

(28)Kurouski, D.; Lombardi, R.A.; Dukor, R.K.; Lednev, I.K.; Nafie, L.A. Direct observation and $\mathrm{pH}$ control of reversed supramolecular Chirality in Insulin Fibrils by Vibrational Circular Dichroism. Chem. Commun. 2010, 46, 7154-7156.

(29)Louzao, I.; Seco, J.M.; Quiñoña, E.; Riguera, R. Control of the Helicity of Poly(phenylacetylene)s: From the Conformation of the Pendant to the Chirality of the Backbone. Angew. Chem. Int. Ed. 2010, 49. 1430-1433.

(30) Neugebauer, J. Induced Chirality in Achiral Media. How Theory Unravels Mysterious Solvent Effects. Angew. Chem. Int. Ed. 2007, 46. 7738-7740.

(31)Debie, E.; Jaspers, L.; Bultinck, P.; Herrebour, W.; Van der Veken, B. Induced Solvent Chirality: A VCD Study of Camphor in $\mathrm{CDCl}_{3}$. Chem. Phys. Lett. 2008, 450, 426-430. 
(32) Yang, G.; Xu, Y. Probing Chiral Solute-Water Hydrogen Bonding Networks by Chirality Transfer Effects: A Vibrational Circular Dichroism Study of Glycidol in Water. J. Chem. Phys. 2009, 130, 164506.

(33)Nicu, V.P.; Neugebauer, J.; Baerends, E.J. Effects of Complex Formation on Vibrational Circular Dichroism Spectra. Chirality 2010, 21, E287-E297. 